\section{Monoclonal Gammopathy of Undetermined Significance: Significant Beyond Hematology}

To the Editor: Monoclonal gammopathies are frequently encountered in clinical practice, and most have so-called undetermined significance. Current diagnostic criteria for monoclonal gammopathy of undetermined significance (MGUS), previously known as benign monoclonal gammopathy, include presence of a monoclonal immunoglobulin in serum of $30 \mathrm{~g} / \mathrm{L}$ or less, $10 \%$ or less of plasma cells in bone marrow, and absence of clinical manifestations related to the $\mathrm{M}$ protein, such as anemia, hypercalcemia, lytic bone lesions, and renal insufficiency. ${ }^{1,2}$ MGUS is found in $3 \%$ of persons older than 50 years, in $5 \%$ of persons older than 70 years, and in up to $7 \%$ of patients seeking medical evaluation. The importance of MGUS lies in its well-known potential to progress to multiple myeloma or a related plasma cell disorder at an average rate of $1 \%$ per year. However, even small and nonprogressive B-cell clones can have profound consequences that may be less familiar to clinicians. We present illustrative cases of probable immune-mediated disorders strongly associated with MGUS. These nonmalignant disorders are rare, and with such disorders patients may present to a variety of medical specialists. Presence of the M protein may be a valuable clue to the diagnosis. This case series is intended to increase awareness of these disorders and their association with M proteins.

\section{Report of Cases and Discussion}

Case 1: Acquired C1 Inhibitor Deficiency. A 60-year-old woman was referred for investigation of recurrent abdominal pain of 1 year's duration. Seven months before presentation, urgent laparoscopy performed elsewhere was unremarkable other than revealing the presence of ascites. Two weeks before referral, another laparoscopic procedure was performed during a bout of intense abdominal pain. Again, ascites was evident. Acute abdominal pain recurred, and the patient was transferred to our hospital. MGUS, IgG $\kappa$ monoclonal protein, had been diagnosed 9 years previously; family history was noncontributory. The patient's abdomen was diffusely tender without rebound tenderness or guarding. Results of all routine laboratory tests, including C-reactive protein levels, were normal. Complement assays showed low levels of CH50, C1q, and $\mathrm{C} 4$, whereas $\mathrm{C} 3$ levels were normal. $\mathrm{C} 1$ inhibitor levels were low, both quantitatively $(0.12 \mathrm{~g} / \mathrm{L}$; reference range, $0.21-0.39$ $\mathrm{g} / \mathrm{L})$ and functionally ( $<26 \%$; reference range, $70 \%-130 \%)$.

Acquired C1 inhibitor deficiency was diagnosed. During a subsequent attack of abdominal pain, computed tomography confirmed bowel wall edema. At last follow-up, 14 years after the diagnosis, the patient was doing well. With administration of $200 \mathrm{mg}$ of danazol, up to 4 times a day, the $\mathrm{C} 1$ inhibitor level and function normalized. Attacks became less frequent and less severe and could be managed with addition of tranexamic acid. The monoclonal gammopathy was not progressive.

Discussion. C1 inhibitor is an inhibitor of the first component of the classic complement pathway and of the kinin-gen-

(C) 2009 Mayo Foundation for Medical Education and Research erating pathways. When the $\mathrm{C} 1$ inhibitor level is deficient, uncontrolled complement activation and release of inflammatory mediators cause angioedema, characterized by capillary hyperpermeability and extravasation of fluid and proteins. ${ }^{3-6}$ Typically, episodes of angioedema recur without urticaria or pruritus. This nonallergic angioedema most often affects the skin, upper airway, and bowel. Symptoms range from minor inconvenience to life-threatening laryngeal edema. Abdominal attacks can cause severe pain, nausea, and vomiting, and unnecessary abdominal surgery may be performed. A shift of fluids into the interstitium or peritoneal cavity during abdominal attacks can cause hemoconcentration and clinically important hypotension. Despite the impressive clinical picture, inflammatory signs are typically absent (eg, normal C-reactive protein level). Most episodes are self-limiting, lasting 1 to 7 days and recurring every 1 to 9 months, often in a uniform pattern. Hereditary angioedema usually presents during the first or second decade of life. ${ }^{6}$ Family history usually points to autosomal dominant inheritance. Hereditary angioedema is due to either a lack of synthesis of the C1 inhibitor ( $85 \%$ of cases) or the synthesis of a nonfunctional protein (15\% of cases). With the hereditary form, no associated disease is found, and $\mathrm{C} 1 \mathrm{q}$ levels are normal.

Acquired C1 inhibitor deficiency is less frequent: 145 cases have been reported since the first description in $1972 .{ }^{7}$ Acquired C1 inhibitor deficiency affects adult or elderly patients with no family history of this deficiency and is often associated with low-grade lymphoproliferative disorders, including MGUS in up to $40 \%$ of cases.$^{3-5,7}$ The M protein can be $\operatorname{IgG}$, IgM, or IgA. The immunoglobulin is hypothesized to mediate consumption and/or inactivation of the $\mathrm{C} 1$ inhibitor. In the acquired form of $\mathrm{C} 1$ inhibitor deficiency, $\mathrm{C} 1 \mathrm{q}$ serum levels are decreased, which is attributed to consumption due to immune complex formation. $\mathrm{C} 4$ levels are low in both the inherited and the acquired form, whereas C3 levels are normal. Management of acquired C1 inhibitor deficiency consists of treating the underlying disorder, if possible, and preventing and treating angioedema. Acute angioedema is treated with antifibrinolytic agents, such as tranexamic acid. These agents have been advocated especially for prophylactic use. ${ }^{8}$ $17-\alpha$-Alkylated androgens (including danazol) have been used prophylactically because androgens increase liver protein synthesis, including $\mathrm{C} 1$ inhibitor levels and function. ${ }^{5}$ Response to $\mathrm{C} 1$ inhibitor substitution or fresh frozen plasma in patients with the acquired form of $\mathrm{C} 1$ inhibitor deficiency varies because of hypercatabolism.

Case 2: Systemic Capillary Leak Syndrome. A 61-yearold man was referred to our hospital because of hypovolemic shock. Hypotension progressed to immeasurable blood pressure levels, and associated edema developed. Within 24 hours, his hemoglobin concentration increased from $15.3 \mathrm{~g} / \mathrm{dL}$ to $22.9 \mathrm{~g} / \mathrm{dL}$ (reference ranges shown parenthetically) (12.0-16.0 $\mathrm{g} / \mathrm{dL}$ ), along with an increase in blood urea nitrogen levels; his serum albumin level decreased from $40 \mathrm{~g} / \mathrm{L}$ to $35 \mathrm{~g} / \mathrm{L}$ (40.2-47.6 g/L). Intense fluid resuscitation was initiated. After a few hours, hemodynamic stabilization was obtained, and a polyuric phase followed. During the 18 months before the index admission, the patient was admitted to a regional hospital 
5 times because of shock. Episodes were variably preceded by malaise, abdominal discomfort, fatigue, rhinitis, palpitations, dyspnea, thirst, oliguria, orthostatic symptoms, fever, and palpebral edema. Duration of attacks ranged from 1 day to several days, and they were characterized by extreme circulatory collapse and high fluid requirements. Between crises, the patient was free of symptoms. A monoclonal $\operatorname{IgG} \kappa$ gammopathy was found and systemic capillary leak syndrome diagnosed. A prophylactic regimen that consisted of terbutaline and theophyllines was initiated.

The patient has been followed up for 9 years. Although the attacks are less severe since the diagnosis, except for 2 occasions that required admission to the intensive care unit, symptoms recur irregularly and are accompanied by weight gain, lowering of blood pressure, and decreased urinary output. At onset of a prodromal phase, methylprednisolone is added for 1 or 2 days to the usual prophylactic regimen. The IgG monoclonal gammopathy remains stable at a low level.

Discussion. The systemic capillary leak syndrome is a rare but life-threatening disorder, characterized by recurrent attacks of hypovolemic shock and generalized edema. ${ }^{9}$ About 100 cases have been reported..$^{10}$ The syndrome is attributed to transient capillary hyperpermeability with extravasation of albumin-rich plasma, up to $70 \%$ of total plasma volume. The acute phase is characterized by a typical triad of hypotension, increased hematocrit, and hypoalbuminemia. Monoclonal gammopathy, usually $\mathrm{IgG}$, is typically present. The pathophysiologic role of the M protein is unclear. Attacks of the systemic capillary leak syndrome vary in frequency, severity, and duration. The systemic capillary leak syndrome affects otherwise healthy middle-aged individuals with no positive family history of this condition. The classic clinical evolution is triphasic. First, a prodromal phase of a few hours to days is characterized by polydipsia and oliguria and by nonspecific symptoms such as those described in the case report. Second, the capillary leak phase, lasting 1 to 4 days, is heralded by generalized edema accompanied by hemoconcentration, hypotension that can lead to hypovolemic shock, and weight gain. Intestinal edema, ascites, pleural and pericardial effusions, muscular edema (with the compartment syndrome), and oliguric nonproteinuric prerenal failure may occur. Third, during the recruitment phase, fluid and proteins are reabsorbed, and polyuria replaces oliguria. In this latter phase, overagressive fluid correction may lead to pulmonary edema.

Supportive treatment consists of judicious volume therapy: fluid substitution in the capillary leak phase and promotion of diuresis of accumulated fluid in the recruitment phase. $\beta_{2}$ Agonists (eg, terbutaline) and theophylline have been recommended as prophylactic therapy, without definite proof of efficacy. ${ }^{11-13}$ Recently, 3 patients were treated successfully with high-dose intravenous immunoglobulins. ${ }^{14}$

Case 3: Acquired von Willebrand Syndrome. A 38-yearold woman with an enlarged left ovary was referred for preoperative evaluation. She noted prolonged bleeding after minor cutaneous injury and menorrhagia of 1 year's duration. No other bleeding symptoms were evident. Previous dental surgery (4 years previously) and knee surgery (25 years pre- viously) were uncomplicated. The patient took no regular medication and had no family history of bleeding. Platelet count, prothrombin time, and D-dimer level were normal. The activated partial thromboplastin time was prolonged. Factor VIII coagulant, von Willebrand antigen, and ristocetin cofactor were profoundly decreased, and platelet aggregation was severely depressed. A monoclonal gammopathy $\operatorname{IgG} \kappa$ was found, without evidence of multiple myeloma. Acquired von Willebrand syndrome was diagnosed. Correction of the hemostatic defect after desmopressin administration was brief. Likewise, the effect of von Willebrand factor concentrate substitution was limited and temporary. Intravenous IgG was infused with total correction for several days, facilitating laparoscopic resection of a benign ovary tumor without complications. During the following years, hip arthroplasty and dental extractions were performed safely with prior administration of intravenous immunoglobulin.

Discussion. The acquired von Willebrand syndrome is an uncommon hemostatic disorder, usually arising in elderly patients with no prior personal or family history of bleeding. Since 1968, more than 300 cases have been reported. ${ }^{15,16}$ Of the various diseases associated with acquired von Willebrand syndrome, hematoproliferative disorders, including MGUS, are most frequent, occurring in $50 \%$ to $60 \%$ of patients. ${ }^{15-17}$ Other disorders associated with the acquired von Willebrand syndrome include hypothyroidism, solid tumors, and autoimmune disorders. Laboratory findings and clinical presentation are the same as in patients with the congenital form of von Willebrand disease. The pathogenesis is unclear; however, autoantibody-mediated inhibition of von Willebrand factor function or enhanced clearance of the factor VIII von Willebrand factor complex is thought to play a role. Management consists of treating the underlying disorder, if possible, and correcting and preventing bleeding episodes. The effects of desmopressin infusion, which induces release of von Willebrand factor from endothelial cells, are transient and less pronounced than in congenital von Willebrand disease, which has been attributed to an accelerated plasma clearance of the von Willebrand factor. Similarly, large doses of von Willebrand factor-containing concentrates may be required to overwhelm an inhibitory autoantibody. Administration of high-dose intravenous $\mathrm{IgG}$ can produce more sustained responses in patients with acquired von Willebrand syndrome, especially when associated with MGUS or lymphoproliferative disorders. ${ }^{15-17}$

Case 4: Schnitzler Syndrome. A 37-year-old man presented with progressive aching of the joints, especially of his wrists and ankles, and morning stiffness. During the past 2.5 years, he had repeatedly been seen at the emergency department because of bouts of fever. Laboratory tests revealed chronic inflammation, also occurring between febrile episodes, with $\mathrm{C}$-reactive protein levels varying between 30 and $94 \mathrm{mg} / \mathrm{L}(<5.0 \mathrm{mg} / \mathrm{L})$ and erythrocyte sedimentation rates higher than $55 \mathrm{~mm} / \mathrm{h}(1-15 \mathrm{~mm} / \mathrm{h})$. Chronic urticaria waxed and waned, with minor itching. Evidence of monoclonal gammopathy $\operatorname{IgM} \kappa$, without evidence of Waldenström disease, led to the diagnosis of Schnitzler syndrome. Bone scintigraphy showed periosteal reaction at both knees. Treatment with 
TABLE. Examples of Probable Immune-Mediated Disorders Strongly Associated With Monoclonal Gammopathy of Undetermined Significance (MGUS)
Primarily dermatological ${ }^{26}$
Schnitzler syndrome $e^{\mathrm{a}, \mathrm{b}}$
Scleromyxedema,
Scleredema
Necrobiotic xanthogranuloma
Plane xanthoma
Primarily neurologic $\mathrm{c}^{27}$
MGUS neuropathy ${ }^{\mathrm{a}}$
Miscellaneous
Type II mixed cryoglobulinemia ${ }^{a}$
Chronic cold agglutinin disease ${ }^{\mathrm{a}}$
Acquired $\mathrm{C} 1$ inhibitor deficiency ${ }^{\mathrm{b}}$
Systemic capillary leak syndrome $\mathrm{e}^{\mathrm{a}, \mathrm{b}}$
Acquired von Willebrand syndrome ${ }^{b}$

\footnotetext{
${ }^{\text {a }}$ Presence of monoclonal protein virtually obligatory.

${ }^{\mathrm{b}}$ Discussed in current series.
}

anakinra, $100 \mathrm{mg} / \mathrm{d}$ subcutaneously, induced prompt resolution of articular and cutaneous symptoms and normalization of the laboratory inflammatory markers but resulted in moderate neutropenia. Temporary interruption of therapy provoked recurrence of symptoms and signs.

Discussion. Schnitzler syndrome, described in 1972, is a rare disorder characterized by a chronic urticarial rash and monoclonal gammopathy, primarily of the IgM type. ${ }^{18}$ Minor criteria include intermittent fever, arthralgia or arthritis, bone pain, lymphadenopathy, hepatomegaly and/or splenomegaly, elevated erythrocyte sedimentation rate and/or leukocytosis, and bone abnormalities. ${ }^{18,19}$ Schnitzler syndrome can be diagnosed when both major criteria and 2 or more minor criteria are fulfilled, and other causes are excluded. Ninety-four patients have been reported as having this syndrome. ${ }^{18}$ Chronic, often nonitching, urticaria is the hallmark and usually the first symptom. Of the various therapeutic options that have been proposed, anakinra, an interleukin 1 receptor antagonist, shows the most promise; it has induced complete remission (with continuous administration) in 7 of 7 previously described patients. ${ }^{18}$

Case 5: Scleromyxedema. A 39-year-old man experienced 4 episodes of confusion during a 2-year period, each predated by a flulike illness and culminating in status epilepticus with prolonged postictal coma. Between episodes, cognitive dysfunction persisted, affecting memory, attention, executive function, and word finding. During the first episode, an M protein $\mathrm{IgG} \kappa$ was found. Neuroimaging and cerebrospinal fluid analysis were unrevealing. The appearance over a few weeks of erythematous plaques on the face and skin creases over the glabella suggested the diagnosis of scleromyxedema, which was confirmed pathologically. The M protein value increased from $7 \mathrm{~g} / \mathrm{L}$ to $26 \mathrm{~g} / \mathrm{L}$ in 1 month's time, anemia developed, and bony plasmocytoma was found; a diagnosis of multiple myeloma was established. Hematologic treatment consisted of plasmapheresis, dexamethasone, intravenous immunoglobulin, autologous stem cell transplant, and thalidomide. Six months after the diagnosis of scleromyxedema, the patient experienced an acute myocardial infarction. During the following year, the patient's condition improved, including sub- stantial recovery of cognitive functions, and his antiepileptic medication was tapered.

Discussion. Scleromyxedema is a rare disease of unknown etiology that is characterized by hyaluronic acid deposition into the superficial dermis and other organs and production of a monoclonal protein, primarily $\operatorname{IgG} \lambda \lambda^{20,21}$ Papular mucinosis and generalized lichen myxedematosus are related terms. Usually, the disease appears equally in either sex and in persons between ages 30 and 50 years. The cutaneous eruption consists of waxy papules, varying from 2 to $5 \mathrm{~mm}$, with marked sclerosis and induration of the skin, especially of the head, neck, and extremities. Involvement of the gastrointestinal tract, musculoskeletal system, renal system, and cardiopulmonary system is common. ${ }^{21}$ Reports of cardiac pathologic findings have included myocardial infarction. Neurologic manifestations include encephalopathy, stroke, seizures, coma, psychosis, and peripheral neuropathies. ${ }^{22}$ As in the current case, patients have been described in whom neurologic abnormalities preceded the classical skin lesions. The term dermato-neuro syndrome has been coined to describe a flulike prodrome, followed by fever (high temperatures), seizures, and coma in the setting of scleromyxedema. ${ }^{21}$ Treatment of scleromyxedema has included a number of modalities, including corticosteroids and immunosuppressive agents, antineoplastic agents, plasmapheresis, thalidomide, and stem cell transplant. Recently, treatment with intravenous immunoglobulin has been associated with high response rates but requires maintenance infusions to preserve disease control. ${ }^{23}$

\section{Conclusion}

This case series highlights the potentially profound clinical importance of a monoclonal protein, besides its capability of malignant transformation. Most of these M protein-related disorders are rare, probably in part due to underrecognition, and some are life-threatening. The mechanisms of these diseases are not fully understood, and the pathogenetic role of the monoclonal protein is uncertain. Immune-mediated mechanisms are commonly implicated. This contrasts with other monoclonal component-related diseases that are caused by M protein aggregation, such as light chain cast nephropathy, AL amyloidosis, light chain deposition disease, crystal-storing histiocytosis, and cryoglobulinemia type I. ${ }^{24,25}$ Examples of probable immune-mediated disorders strongly associated with MGUS are listed in the Table. The disorders with primary dermatological and neurologic manifestations have been reviewed. ${ }^{26,27} \mathrm{~A}$ monoclonal protein is almost a sine qua non in certain disorders (such as the systemic capillary leak syndrome, scleromyxedema, and Schnitzler syndrome), whereas the underlying diseases can be more variable in other disorders (such as acquired von Willebrand syndrome and acquired $\mathrm{C} 1$ inhibitor deficiency).

The diagnosis of acquired C1-inhibitor deficiency should be suspected in patients with repetitive, often stereotypic episodes of angioedema, with no urticaria or pruritus and no family history, as well as in patients with recurrent acute abdomen with normal C-reactive protein levels and low $\mathrm{C} 4$ levels. The systemic capillary leak syndrome should be suspected in 
patients with repetitive hypovolemic shock, with capillary leak and hemoconcentration. Acquired von Willebrand syndrome is suggested by mucocutaneous or postoperative bleeding due to a primary hemostatic defect in patients with no family history of bleeding. Schnitzler syndrome is heralded by repetitive fevers and chronic, often initially nonpruritic, urticaria. Scleromyxedema is characterized by a typical skin eruption and visceral or neurologic involvement. In all these situations, knowledge of the disorders and identification of a monoclonal peak may pave the way to the correct diagnosis. The $\mathrm{M}$ protein is mostly of the IgG type, although IgM may predominate, as in the typical Schnitzler syndrome. The likelihood of progression to a malignant cell disorder is thought to be similar to that in persons with a "clincally silent" MGUS.

Increased recognition of these disorders may facilitate the corroboration of the pathogenetic role of the $\mathrm{M}$ protein and lead to more specific or ameliorated therapies. Thus, MGUS is not always innocent or benign and can be of utmost importance.

Steven Vanderschueren, MD, PhD

Marieke Mylle, MD

Daan Dierickx, MD

Michel Delforge, MD, PhD

Peter Verhamme, MD, PhD

Kathelijne Peerlinck, MD, PhD

Wouter Meersseman, MD

Daniël C. Knockaert, MD, PhD

University Hospital Gasthuisberg Leuven, Belgium

1. Bladé J. Monoclonal gammopathy of undetermined significance. $N$ Engl J Med. 2006;355(26):2765-2770.

2. Kyle RA, Rajkumar SV. Monoclonal gammopathy of undetermined significance. Br J Haematol. 2006;134(6):573-589.

3. Agostoni A, Aygören-Pürsün E, Binkley KE, et al. Hereditary and acquired angioedema: problems and progress: Proceedings of the Third C1 Esterase Inhibitor Deficiency Workshop and Beyond. J Allergy Clin Immunol. 2004;114(3)(suppl):S51-S131.

4. Rosen FS, Davis AE III. Deficiencies of $\mathrm{C} 1$ inhibitor. Best Pract Res Clin Gastroenterol. 2005;19(2):251-261.

5. Markovic SN, Inwards DJ, Frigas EA, Phyliky RP. Acquired C1 esterase inhibitor deficiency. Ann Intern Med. 2000;132(2):144-150.

6. Zuraw BL. Hereditary angioedema. N Engl J Med. 2008;359(10):10271036.

7. Castelli R, Deliliers DL, Zingale LC, Pogliani EM, Cicardi M. Lymphoproliferative disease and acquired $\mathrm{C} 1$ inhibitor deficiency [letter]. Haematologica. 2007;92(5):716-718.

8. Cicardi M, Zingale LC, Pappalardo E, Folcioni A, Agostoni A. Autoantibodies and lymphoproliferative diseases in acquired $\mathrm{C} 1$-inhibitor deficiencies. Medicine (Baltimore). 2003;82(4):274-281.

9. Clarkson B, Thompson D, Horwith M, Luckey EH. Cyclical edema and shock due to increased capillary permeability. Am J Med. 1960 Aug; 29:193-216.

10. Dhir V, Arya V, Malav IC, Suryanarayanan BS, Gupta R, Dey AB. Idiopathic systemic capillary leak syndrome (SCLS): case report and systematic review of cases reported in the last 16 years. Intern Med. 2007;46(12):899-904. Epub 2007 Jun 15.

11. Amoura Z, Papo T, Ninet J, et al. Systemic capillary leak syndrome: report on 13 patients with special focus on course and treatment. Am J Med. 1997;103(6):514-519.

12. Droder RM, Kyle RA, Greipp PR. Control of systemic capillary leak syndrome with aminophylline and terbutaline. Am J Med. 1992;92(5): 523-526.

13. Tahirkheli NK, Greipp PR. Treatment of the systemic capillary leak syndrome with terbutaline and theophylline: a case series. Ann Intern Med. 1999;130(11):905-909.
14. Lambert M, Launay D, Hachulla E, et al. High-dose intravenous immunoglobulins dramatically reverse systemic capillary leak syndrome. Crit Care Med. 2008;36(7):2184-2187.

15. Kumar S, Pruthi RK, Nichols WL. Acquired von Willebrand disease. Mayo Clin Proc. 2002;77(2):181-187.

16. Franchini M, Lippi G. Acquired von Willebrand syndrome: an update. Am J Hematol. 2007;82(5):368-375.

17. Frederici AB. Acquired von Willebrand syndrome: an underdiagnosed and misdiagnosed bleeding complication in patients with lymphoproliferative and myeloproliferative disorders. Semin Hematol. 2006;43(1)(suppl 1): S48-S58.

18. de Koning HD, Bodar EJ, van der Meer JW, Simon A; Schnitzler Syndrome Study Group. Schnitzler syndrome: beyond the case reports: review and follow-up of 94 patients with an emphasis on prognosis and treatment. Semin Arthritis Rheum. 2007 Dec;37(3):137-148. Epub 2007 Jun 21.

19. Lipsker D, Veran Y, Grunenberger F, Cribier B, Heid E, Grosshans E. The Schnitzler syndrome: four new cases and review of the literature. Medicine (Baltimore). 2001;80(1):37-44

20. Gabriel SE, Perry HO, Oleson GB, Bowles CA. Scleromyxedema: a scleroderma-like disorder with systemic manifestations. Medicine (Baltimore). 1988;67(1):58-65.

21. Pomann JJ, Rudner EJ. Scleromyxedema revisited. Int J Dermatol. 2003; 42(1):31-35

22. Berger JR, Dobbs MR, Terhune MH, Maragos WF. The neurologic complications of scleromyxedema. Medicine (Baltimore). 2001;80(5):313-319.

23. Blum M, Wigley FM, Hummers LK. Scleromyxedema: a case series highlighting long-term outcomes of treatment with intravenous immunoglobulin (IVIG). Medicine (Baltimore). 2008;87(1):10-20.

24. Merlini G, Stone MJ. Dangerous small B-cell clones. Blood. 2006 Oct 15;108(8):2520-2530. Epub 2006 Jun 22.

25. Fain O. Monoclonal gammopathies [in French]. Rev Prat. 2006;56(1): 40-50.

26. Daoud MZ, Lust JA, Kyle RA, Pittelkow MR. Monoclonal gammopathies and associated skin disorders. J Am Acad Dermatol. 1999;40(4):507-535.

27. Ropper AH, Gorson KC. Neuropathies associated with paraproteinemia. N Engl J Med. 1998;338(22):1601-1607.

\section{Apical Ballooning Syndrome After Administration of Intravenous Epinephrine During an Anaphylactic Reaction}

To the Editor: First described in Japan, apical ballooning syndrome (ABS), or Takotsubo cardiomyopathy, is an acquired, reversible cardiomyopathy. Catecholamine-induced myocardial stunning is the leading hypothesis for its pathophysiology. ${ }^{1}$

Epinephrine is the treatment of choice for patients with anaphylaxis. ${ }^{2}$ Prompt intramuscular administration is recommended because adverse reactions are more likely with intravenous dosing. ${ }^{3}$ We describe a case of a woman who developed ABS after intravenous administration of epinephrine.

A 41-year-old woman developed itching, hives, lip and tongue swelling, and shortness of breath after a bee sting. After self-administration of diphenhydramine, she presented to a local emergency department. Her vital signs were as follows: blood pressure, 116/93 $\mathrm{mm} \mathrm{Hg}$; pulse, 98 beats/min; respiratory rate, 24 breaths/min; oxygen saturation, 98\%; and temperature, $36.7^{\circ} \mathrm{C}$. She was given intravenous fluids and diphenhydramine. Oral edema appeared to be increasing. Records from the referring emergency department indicate that the physician ordered $0.5 \mathrm{~mL}$ of intravenous epinephrine $(1: 10,000)$, but nursing notes indicate that $0.5 \mathrm{mg}(1: 10,000)$ of epinephrine was administered intravenously. The patient became hypotensive. The physician ordered another $0.5-\mathrm{mL}$ dose of intravenous epinephrine (1:10,000). Again, nursing 


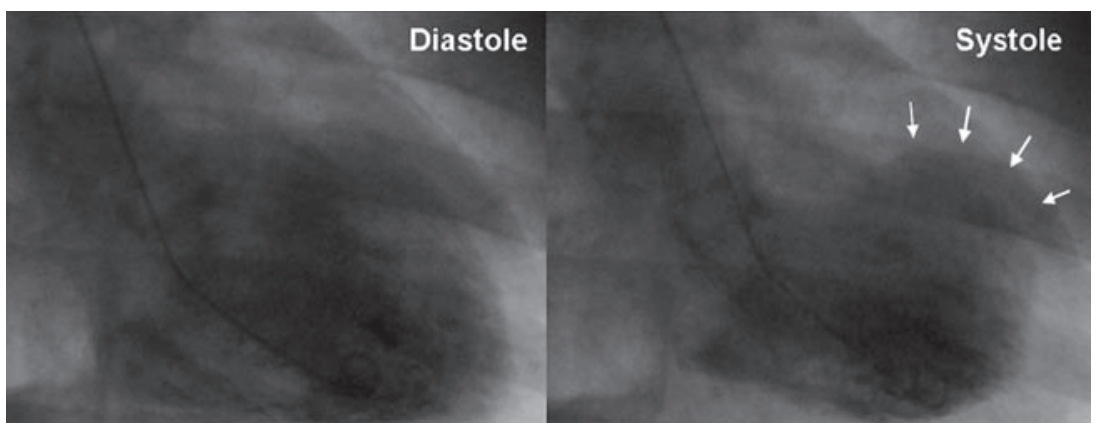

FIGURE. Diastolic and systolic freeze frames from a left ventriculogram of the patient demonstrating basal contraction but akinesis of the anterolateral wall (arrows).

records indicate that a 0.5 -mg dose $(1: 10,000)$ of intravenous epinephrine was administered instead. The patient developed chest pain and wide complex tachycardia. Electrocardiography revealed ST-segment elevation in leads I and aVL and STsegment depression in leads III and aVF, consistent with myocardial infarction. While still receiving nitroglycerin therapy, the patient was transferred to a tertiary center (Mayo Clinic). On arrival, she was treated with dexamethasone and antihistamines. She had a troponin T level of $0.49 \mathrm{ng} / \mathrm{mL}$ (reference ranges provided parenthetically) $(\leq 0.01 \mathrm{ng} / \mathrm{mL})$ and a creatine kinase-MB level of $11.4 \mathrm{ng} / \mathrm{mL}(\leq 6.2 \mathrm{ng} / \mathrm{mL})$.

Cardiac angiography revealed normal coronary arteries. Left ventriculography showed akinesis of posterolateral, lateral, anterolateral, diaphragmatic, and basal septal segments of the left ventricle (ejection fraction, 48\%) (Figure). Followup echocardiography after 22 days showed less extensive wall motion abnormalities and an ejection fraction of $60 \%$.

Testing for IgE yellow jacket venom was positive $(1.08 \mathrm{kU} / \mathrm{L}$ $[<0.35 \mathrm{kU} / \mathrm{L}]$ ). Catechol $O$-methyltransferase genotype testing showed that the patient was a heterozygote and an intermediate metabolizer. Skin tests showed positive reactions to wasp, yellow jacket, yellow hornet, and white-faced hornet venom.

The clinical presentation of our patient met the Mayo Clinic ABS diagnostic criteria. ${ }^{1}$ To our knowledge, this case is the first to implicate intravenous epinephrine administration during anaphylaxis as a cause of ABS.

Intravenous epinephrine is indicated in patients with severe hypotension or cardiac arrest unresponsive to intramuscular epinephrine and fluid resuscitation. A $0.2 \mu \mathrm{g} / \mathrm{kg}$ intravenous bolus is recommended for hypotension and a $0.1-$ to 0.5 -mg dose for cardiovascular collapse. ${ }^{4}$ This patient's hypotension appeared to occur after the first dose of epinephrine. The hypotension likely represented the initiation of adverse cardiac response to epinephrine. The temporal relationship between administration of epinephrine and onset of findings consistent with ABS supports the hypothesis of catecholamine-induced myocardial stunning as the mechanism for left ventricular dysfunction. The patient's catechol $O$-methyltransferase genotype may have also increased her predisposition to ABS.

Our case illustrates that administration of intravenous epinephrine, especially at high doses, may be a trigger for ABS and underscores the risk of inappropriate epinephrine dosing during anaphylaxis. ${ }^{5}$

\author{
Veena Manivannan, MBBS \\ James T. C. Li, MD, PhD \\ Abhiram Prasad, MD, FRCP \\ Ronna L. Campbell, MD, PhD \\ Mayo Clinic \\ Rochester, MN
}

1. Prasad A, Lerman A, Rihal CS. Apical ballooning syndrome (Tako-Tsubo or stress cardiomyopathy): a mimic of acute myocardial infarction. Am Heart J. 2008;155(3):408-417. Epub 2008 Jan 31.

2. Kemp SF, Lockey RF, Simons FER; World Allergy Organization ad hoc Committee on Epinephrine Anaphylaxis. Epinephrine: the drug of choice for anaphylaxis: a statement of the World Allergy Organization. Allergy. 2008;63(8):1061-1070.

3. Pumphrey RS. Lessons for management of anaphylaxis from a study of fatal reactions. Clin Exp Allergy. 2000;30(8):1144-1150.

4. Sampson HA, MuÑoz-Furlong A, Campbell RL, et al. Second symposium on the definition and management of anaphylaxis: summary report-Second National Institute of Allergy and Infectious Disease/Food Allergy and Anaphylaxis Network symposium. J Allergy Clin Immunol. 2006;117(2):391-397. 5. Abraham J, Mudd JO, Kapur NK, Klein K, Champion HC, Wittstein IS. Stress cardiomyopathy after intravenous administration of catecholamines and beta-receptor agonists. J Am Coll Cardiol. 2009;53(15):1320-1325.

\section{Drug-Induced Compulsive Behaviors: Exceptions to the Rule}

To the Editor: We are intrigued by the recent article by Bostwick et $\mathrm{al}^{1}$ on the emergence of impulse control behaviors after treatment with dopamine agonists. It is amazing how these gambling and hypersexuality adverse effects occur and then seem to immediately abate simply by discontinuing the Parkinson disease medication without need for any additional addiction-related treatment. Ropinirole was recently investigated in a phase 4 clinical trial as a treatment for sexual dysfunction secondary to antidepressant pharmacotherapy, ${ }^{2}$ which speaks to its possible effects on sexual behavior. Dopamine agonists may affect the mesolimbic pathway, as do drugs like cocaine and methamphetamine, whose behavioral properties are attributed to this dopaminergic activity; sexual compulsivity is a well-established adverse effect of these medications. ${ }^{3}$ Furthermore, dopamine dysregulation syndrome, an addictive use pattern of dopamine agonists, causes the same behaviors observed in cocaine-depen- 
dent patients, such as punding, a stereotypical motor behavior characterized by an intense fascination with the repetitive handling and examination of objects, particularly mechanical ones. Examples of punding include picking at oneself, taking apart watches and radios, or sorting and arranging common objects (eg, lining up pebbles, rocks, or other small objects). People engaging in punding find immersion in such activities comforting, even when they serve no purpose, and generally feel frustrated when diverted from them. Punding is thought to be related to dopamine use and has been observed in (meth)amphetamine and cocaine users, as well as in some patients with Parkinson disease, gambling addictions, and hypersexuality. ${ }^{4}$

We have questioned whether drugs like cocaine precipitate these psychiatric issues or whether they preexist the drug use. Robinson and Berridge ${ }^{5}$ suggest that prior use of certain drugs sensitizes the mesolimbic reward pathways, producing neuroplastic effects on the brain and increasing the likelihood of future abuse/dependence. Because impulse control disorders such as pathologic gambling and sexual compulsivity are associated with dysfunction in the same neural circuitry, we propose that use of these substances may directly activate these pathways (precipitating acute symptoms of compulsive gambling and/or hypersexuality) and over time may produce neuroplastic changes in these pathways (leading to chronic impulse control behaviors). With cocaine and other dopaminergic-stimulating medications, there may be a temporal use interval necessary to produce adaptive synaptic changes in the mesolimbic system of patients using these substances, and future studies should focus on the timeline of drug use.

In addition, we have treated hundreds of cocaine abusers with comorbid gambling addiction and/or hypersexuality for whom behavioral remission was not automatic once they stopped using cocaine. In fact, most of our patients need longterm treatment in addition to discontinuation of the substance. It would be useful to study the differences in cocaine-induced gambling and/or hypersexuality and dopamine agonist-induced behaviors. A long-term follow-up of the patients in the study by Bostwick et $\mathrm{al}^{1}$ would also be useful to determine whether true behavioral remission was achieved.

Noni A. Graham, MPH

Christopher J. Hammond, MD

Mark S. Gold, MD

University of Florida College of Medicine Gainesville

1. Bostwick JM, Hecksel KA, Stevens SR, Bower JH, Ahlskog JE. Frequency of new-onset pathologic compulsive gambling or hypersexuality after drug treatment of idiopathic Parkinson disease. Mayo Clin Proc. 2009;84(4):310-316.

2. Hellerstein DJ. Treating sexual dysfunction from selective serotonin reuptake inhibitor (SSRI) medication: a study comparing Requip CR to placebo. http://www.clinicaltrials.gov/ct2/show/study/NCT00334048. Accessed August 4, 2009

3. Giovannoni G, O'Sullivan JD, Turner K, Manson AJ, Lees AJ. Hedonistic homeostatic dysregulation in patients with Parkinson's disease on dopamine replacement therapies. J Neurol Neurosurg Psychiatry. 2000;68(4):423-428.

4. O'Sullivan SS, Evans AH, Lees AJ. Dopamine dysregulation syndrome: an overview of its epidemiology, mechanisms and management. CNS Drugs. 2009;23(2):157-170.

5. Robinson TE, Berridge KC. Addiction. Annu Rev Psychol. 2003;54:25-53. Epub 2002 Jun 10.
In reply: We very much appreciate the thoughtful questions posed by Graham et al regarding dopamine-agonist-induced compulsive pathologic behaviors. Although previous studies (including several from our institution) have documented this association, our study is unique in that the frequency was assessed in a community sample, rather than among referral center patients. Moreover, retrospectively relying on the recorded history of these behaviors makes it likely that these are conservative estimates, as we discussed in the article.

The behavioral remission with drug discontinuation in our agonist-treated patients apparently contrasts with the experience of Graham et al among cocaine and methamphetamine addicts, suggesting that different pharmacologic factors may be operative. As we emphasized in our article, available dopamine agonists have a focused and striking affinity for D3 receptors. ${ }^{1}$ In contrast, cocaine and methamphetamine release dopamine and block reuptake, broadly enhancing dopaminergic neurotransmission at all dopamine receptors. Cocaine and methamphetamine also elicit a more pervasive behavioral syndrome, including hyperarousal, hypervigilance, and insomnia, which is distinct from that sometimes provoked by dopamine agonists.

The increasing number of reports documenting these pathologic behaviors among dopamine agonist-treated patients, but rarely among patients treated with carbidopa-levodopa, affirms the association with this drug class. Moreover, similar behavioral syndromes have now been reported in patients with restless legs syndrome who have been administered dopamine agonists, providing further support that the behaviors may be specific to the drugs, rather than to the disease. We advise close monitoring in the weeks after initiating dopamine agonists so that compulsive behaviors can be uncovered and medication tapered or discontinued as appropriate.

J. Michael Bostwick, MD

J. Eric Ahlskog, MD, PhD Mayo Clinic

Rochester, MN

1. Gerlach M, Double K, Arzberger T, Leblhuber F, Tatschner T, Riederer P. Dopamine receptor agonists in current clinical use: comparative dopamine receptor binding profiles defined in the human striatum. J Neural Transm. 2003;110(10):1119-1127.

\section{CORRECTION}

Incorrect data: In the article by Heffner and Klein entitled "Recent Advances in the Diagnosis and Management of Malignant Pleural Effusions," published in the February 2008 issue of Mayo Clinic Proceedings (Mayo Clin Proc. 2008;83(2):235-250), data in the first complete sentence on page 238, left-hand column, were transposed. The sentence should read as follows: Each of these findings has a reported specificity of between $88 \%$ and $100 \%$ and a sensitivity of between $22 \%$ and $56 \%$." 